\title{
Evaluación del Egreso de la Licenciatura en Actividad Física y Deportes de la UABC: Un Ejercicio Comprensivo
}

\author{
Graduation Evaluation of the BA in Physical Activity and Sports \\ of the UABC: A Comprehensive Achievement
}

\author{
José Alfonso Jiménez Moreno * \\ Juan Pablo Machado Parra \\ Joaquín Caso Niebla \\ Emilio Manuel Arrayales Millán
}

Universidad Autónoma de Baja California, México

\begin{abstract}
Con la intención de contar con información que permita retroalimentar el programa educativo de la Licenciatura en Actividad Física y Deportes de la Universidad Autónoma de Baja California se realizó un ejercicio de evaluación al egreso. A través de un enfoque de evaluación comprensiva se diseñaron dos instrumentos. El primero de ellos enfocado en identificar los conocimientos y habilidades profesionales de los egresados en concordancia con el currículum del programa educativo en cuestión, y, el otro, centrado en conocer las habilidades interpersonales relacionadas con un egreso exitoso de este nivel de estudios. Se muestran los resultados psicométricos de los instrumentos, así como los resultados generales de los participantes en los diferentes campus de la universidad. Se concluye la relevancia de los resultados como un insumo para procesos de planeación didáctica y mejora curricular de la Licenciatura en Actividad Física y Deportes; así como la importancia del enfoque de evaluación utilizado, el cual antepone la participación de la comunidad académica en la conformación de la información generada, así como los alcances y limitaciones de una evaluación de este tipo.
\end{abstract}

Descriptores: Evaluación del estudiante; Examen escrito; Evaluación formativa; Formación profesional superior; Educación física.

With the intention of having information to support evaluation of the educational program of the Bachelor Degree on Physical Activity and Sports of the Autonomous University of Baja California an assessment exercise was performed at the end of the undergraduate education. Through a comprehensive evaluation approach, two instruments were designed. The first of them focused on identifying the knowledge and professional skills of the graduates in accordance with the curriculum of the educational program in question, and the other, focused on identifying the interpersonal skills related to a successful exit from this level of studies. The article shows psychometric results of the instruments, as well as the general results of the participants in the different campuses of the university. Conclusions addresses the relevance of the results as an input for didactic planning processes and curricular improvement of the Degree in Physical Activity and Sports. As well, the article addresses the importance of the evaluation approach used, which highlights the participation of the academic community in the formation of the information generated, as well as the scope and limitations of an evaluation of this type.

Keywords: Student evaluation; Written exam; Formative evaluation; Professional training; Physical education.

*Contacto:

jose.alfonso.jimenez.moreno@uabc.edu.mx

ISSN: $1696-4713$

www.rinace.net/reice/

revistas.uam.es/reice
Recibido: $\quad 19$ de enero 2019

$1^{\text {a }}$ Evaluación: 22 de febrero 2019

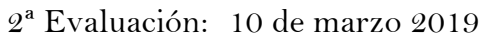

Aceptado: 17 de marzo 2019 


\section{Introducción}

La Universidad Autónoma de Baja California (UABC) es una de las Instituciones de Educación Superior públicas más grandes en el noroeste de México. Dentro de sus objetivos principales se encuentra orientar la formación profesional de sus estudiantes en la solución de necesidades regionales, nacionales e internacionales en todas las áreas del conocimiento en la cual centra su formación (UABC, 2018). Una de las formas de asegurar esta intención es a través de la implementación de mecanismos de evaluación enfocados en las habilidades consideradas como mínimas para el ejercicio profesional esperado (UABC, 2015).

Bajo este contexto, la universidad ha hecho uso de diversos instrumentos externos para valorar los avances y el desempeño de sus estudiantes en los diversos programas educativos de pregrado, particularmente hace uso de los Exámenes Generales para el Egreso de la Licenciatura (EGEL), realizados por el Centro Nacional de Evaluación para la Educación Superior (Ceneval). Sin embargo, existe la preocupación por el hecho que 25 programas educativos $(40 \%$ del total de 63 ) no cuentan con mecanismos de evaluación de la formación de estudiantes al egreso de nivel licenciatura; además que, los aplicados por Ceneval, al no ser curriculares, no necesariamente atienden a las necesidades de los programas educativos al querer considerar sus resultados como un insumo para la orientación curricular (Jiménez, 2017). Uno de los programas de la UABC que no contaba con mecanismos de valoración del egreso como insumo de retroalimentación es la Licenciatura en Actividad Física y Deportes (LAFYD), que se oferta en la Facultad de Deportes (FD) en los tres campus universitarios: Mexicali, Tijuana y Baja California.

Con la finalidad de contar con un mecanismo de evaluación de conocimientos y habilidades al egreso de la formación profesional en actividad física y deporte, la FD, preocupada por establecer formas de retroalimentación de su programa de licenciatura, tuvo un acercamiento con el Instituto de Investigación y Desarrollo Educativo (IIDE), instancia universitaria que fungió como orientador metodológico para el desarrollo de este emprendimiento. Bajo esta intención, ambas instancias desarrollaron en conjunto un ejercicio de evaluación del egreso de sus estudiantes mediante la elaboración de dos instrumentos. El primero de ellos orientado a valorar las habilidades de los egresados relacionadas con las competencias enunciadas en el perfil de egreso de la LAFYD (UABC, 2017). Por su parte, el segundo es un cuestionario dirigido a estudiantes para conocer sus habilidades intra e interpersonales relacionadas con un egreso exitoso (National Academies of Sciences, Engineering and Medicine, 2017). El mayor valor de este ejercicio recae en la intención de retratar una realidad educativa en particular -en este caso, el egreso de los estudiantes de la LAFYD- a partir del perfil profesional esperado por la Facultad de Deportes, con la participación de más de 70 de sus académicos, quienes depositaron su experiencia laboral y docente en la intención de retroalimentar al programa educativo al cual dan vida de forma cotidiana.

Este ejercicio de evaluación representa un ejercicio inédito dentro de la UABC, como el primer programa educativo en realizar un ejercicio de evaluación del egreso de esta naturaleza; de la misma manera lo es en la formación en actividad física en México. 


\section{Enfoque de la evaluación}

Al ser una de las 34 universidad públicas estatales de México, la UABC tiene la responsabilidad de cumplir con funciones de docencia, generación y aplicación innovadora del conocimiento, extensión y difusión de la cultura (SEP, 2018). En estas acciones, la UABC se interesa por asegurar que, al finalizar la formación profesional, sus egresados hayan adquirido y desarrollado habilidades y conocimientos que son importantes para su ejercicio profesional. El ejercicio tuvo como base la evaluación del egreso, pero no solo con el fin de emitir puntajes en una prueba, sino como una manera de reflejar los conocimientos y habilidades esperados dentro del propio programa educativo a través de mecanismos construidos por la propia comunidad académica encargada de la formación de los próximos profesionistas. En este sentido, el programa de licenciatura en cuestión fue el centro del ejercicio de la evaluación, de manera que fueron sus condiciones contextuales y las competencias enunciadas en el Plan de estudios lo que delimitó el ejercicio desarrollado.

Para el desarrollo de esta tarea se procuró realizar un ejercicio que no basara su eje meramente en lo psicométrico, procurando trascender la aplicación del instrumento (Mateo, 2006). Dado que se pretende generar información que funja como un insumo para retroalimentar la formación promovida por el programa educativo en cuestión, se asumió que los instrumentos construidos solo reflejarían un fragmento de toda la complejidad que representa la formación profesional, ello en concordancia con la perspectiva de evaluación comprensiva propuesta por Stake (2006). El modelo comprensivo implica que los ejercicios de evaluación comuniquen la experiencia que las personas viven dentro del programa de interés. Para ello es crucial que el eje de la propuesta no sea la medición en sí misma, sino que los instrumentos y datos resultantes reflejen la diversidad de perspectivas que constituyen el fenómeno de interés, en este caso, la complejidad del egreso profesional. De acuerdo con Stake (2006), la evaluación comprensiva debe tomar en cuenta los intereses y preocupaciones de todos aquellos que estén involucrados, debido a que la diversidad de perspectivas es lo que permite conformar una realidad implicada en los procesos educativos.

En ese sentido, fue crucial considerar la limitante epistemológica que el método establece para la interpretación de los resultados y la posterior retroalimentación de la formación de los estudiantes de la LAFYD (Jiménez, 2014). De tal suerte que, incluso previo al desarrollo de instrumentos se asumió que el ejercicio de evaluación no reflejaría la totalidad de la posibilidad de ejercicio profesional de los egresados de la LAFYD, sino solo aquellos que se lograsen construir en conjunto con la comunidad académica de la FD, además de las limitantes epistemológicas que los instrumentos en sí mismos delimitan (Jiménez, en prensa; Santos Guerra, 1988).

El modelo de Stake sugiere que la evaluación necesita desarrollarse con diferentes audiencias y responder a sus necesidades evaluativas para facilitar la toma de decisiones. No centra tanto su atención a elementos de diseño riguroso de instrumentos, sino en atender a las necesidades e intereses de los stakeholders para poder proveer información que sea de utilidad para el programa de interés. Bajo la perspectiva comprensiva, un evaluador no debe juzgar, sino diseñar estrategias que permitan recoger los juicios de las personas relacionadas con el programa educativo con el fin de proporcionar información que facilite la toma de decisiones. De esta manera, la evaluación del egreso de la LAFYD de la UABC se desarrolló bajo una postura que se aleja de considerarse como un elemento 
únicamente técnico, cuyo interés predominante sea el diseño metodológico, sino como un proceso de diálogo con la comunidad académica de la FD con el fin de identificar los elementos de interés sobre el egreso de sus estudiantes.

\section{Método}

El desarrollo de un ejercicio como este implica necesariamente la consideración de técnicas de recolección de información. Tomando en cuenta la intención de recabar información de todos los egresados del programa en los tres campus universitarios, se pensó en el uso de dos instrumentos: (1) una prueba orientada hacia la identificación de las habilidades relacionadas con el ejercicio profesional de la LAFYD; y (2) un cuestionario orientado a identificar elementos intra e interpersonales en los estudiantes que, de acuerdo con la literatura, influyen en los resultados de la formación profesional. A continuación se describe el proceso de construcción de cada instrumento.

a) Prueba para valorar los conocimientos y habilidades de los egresados

El interés de esta evaluación se centra en emitir una retroalimentación de la formación de los próximos profesionistas en actividad física y deporte, teniendo en consideración un contexto diverso, así como una realidad intersubjetiva que influye, sin duda, en los resultados de egreso. En este marco de evaluación se consideró necesaria la participación de agentes del programa educativo, no bajo un papel de expertos en medición de rasgos del aprendizaje, sino como colaboradores clave para sustentar el diseño metodológico que guio la elaboración del instrumento de evaluación. Bajo el enfoque comprensivo (Stake, 2006) se promovió el trabajo colaborativo, en el cual los actores propios de la LAFYD participaron en la definición de elementos por evaluar, así como en la delimitación de aspectos de interés por reflejar en el instrumento elaborado.

Para desarrollar las diferentes actividades asociadas a la tarea evaluativa, se conformaron tres comités en los que se involucraron más de 70 docentes participantes del proyecto. A continuación, se describen las actividades realizadas por los comités:

1. Definición del marco de evaluación: este ejercicio se desarrolló bajo una estrategia universal de definición de constructos, estableciendo las dimensiones, acciones y habilidades concretas que permitieran la construcción de ítems o reactivos ${ }^{1}$. El insumo principal de este ejercicio fueron las competencias que cada asignatura abona al perfil de egreso. A partir del perfil de egreso, los docentes identificaron las competencias que se desarrollan en el aula y la importancia relativa de cada contenido, lo que permitió establecer cinco áreas o competencias por evaluar y los pesos específicos de cada una con base en su contribución a dicho perfil.

El ejercicio de delimitación del constructo implicó no solo la definición de las áreas de la prueba -que representan las áreas de ejercicio profesional fomentadas por el programa de estudios de la LAFYD-, sino también su decantación, con fines de delimitar de forma precisa la información que se pretende recolectar para los fines de esta evaluación. Si bien es imposible la observación de toda la posibilidad de acciones y habilidades relacionadas con el ejercicio profesional de quien estudia la LAFYD, el ejercicio de delimitación de

${ }^{1}$ En este manuscrito se utilizan de forma indistinta los términos "ítems" y "reactivos" para referir a las formas de medida de habilidades y aprendizajes concretos. 
especificaciones permite recabar una muestra de estas habilidades con fines de alcanzar el objetivo de la evaluación (Webb, 2012). En este sentido, fueron los docentes de la FD quienes establecieron esta muestra de habilidades por medir, resaltando así la relevancia del programa educativo al que esta evaluación pretende dar respuesta, así como la perspectiva de los actores, que son quienes determinan la actividad formadora de la LAFYD.

La dinámica para la definición de los elementos por evaluar se estableció a partir de dos reuniones realizadas entre agosto y septiembre del 2017, en las cuales un grupo de 15 docentes de la FD definieron las áreas de la prueba, así como las habilidades que conforman cada una de ellas. Para esta definición, la comisión de académicos fundamentó su trabajo en la pregunta: ¿Qué tiene que hacer un egresado de la LAFYD para ejecutar las competencias esperadas en el perfil de egreso? Considerando como insumo principal las competencias enunciadas en el perfil de egreso de la LAFYD ${ }^{2}$. Como principios orientadores en esta definición se consideró:

- Iniciar con verbo en presente.

- Redactarlo en términos observables: "Diagnostica las necesidades de formación en educación física de una escuela u organización de un nivel educativo en particular", por ejemplo.

- Se consideraron como referencia los contenidos temáticos de las asignaturas que abonan a cada una de las competencias establecidas en el perfil de egreso ${ }^{3}$.

- El conjunto de las acciones enlistadas debía englobar la competencia profesional esperada.

- En caso de ser necesario, se podría incluir en la prosa algunos valores estipulados en la competencia (objetividad, respeto, tolerancia, etc.).

A este primer nivel de desagregación de las competencias profesionales esperadas en el perfil de egreso se les denominó Acciones. Una vez definidas las Acciones, se consideró necesario establecer un nivel menor de reducción del constructo, al cual se le denominó Habilidades. Para su identificación el grupo de académicos participantes consideró la pregunta eje: ¿Cuáles son las habilidades involucradas en las acciones enlistadas que permiten valorar su ejecución? La delimitación de Habilidades, al igual que con las Acciones, consideró:

- Redactarse con verbo en presente.

- Utilizar un verbo observable (evitar: "analizar", "reflexionar").

- Evitar verbos enfocados en el conocimiento conceptual o memorístico (por ejemplo, "conoce").

- Los PUa que abonan a la competencia.

- El conjunto de las habilidades enlistadas debía englobar la acción esperada.

\footnotetext{
2 Pueden revisarse a detalle en: http://deportes.uabc.mx/index.php/programas-educativos/licenciatura-en-actividadfisica/egreso

${ }^{3}$ En UABC, el formato que incluye la descripción de las asignaturas se le suele denominar "Programa de Unidad de Aprendizaje" (PUA). Los PUA de la LAFyD pueden consultarse en: http://deportes.uabc.mx/index.php/programaseducativos/licenciatura-en-actividad-fisica/plan-2012-2/u-aprendizaje-2012
} 
- Si se valoraba necesario, se podrían incluir en la prosa los valores estipulados en la competencia (objetividad, respeto, tolerancia, etc.).

Para cada Habilidad enlistada se señalaba el tipo de complejidad por valorar, de acuerdo con la propuesta de Bloom Revisada (Krathwohl, 2002), evitando los valores extremos (recordar y crear) en función de la limitación que el uso de un instrumento de opción múltiple representa para la medición del nivel más alto de esta taxonomía, así como evitar el uso de reactivos meramente conceptuales. De esta manera, el aprendizaje evaluado se concentró en las siguientes complejidades cognitivas: comprensión, aplicación, análisis o evaluación. El establecimiento del nivel de complejidad cognitiva para cada Habilidad permitió asegurar la medición de habilidades de forma sistematizada, independientemente de quien desarrollara el reactivo.

El resultado de este ejercicio permitió establecer el marco de las habilidades por evaluar con base en la agrupación de cinco áreas de ejercicio profesional del egresado de la LAFYD, las cuales están establecidas en el perfil de egreso del programa. Las áreas o competencias establecidas se muestran en cuadro 1.

Cuadro 1. Áreas del examen para la evaluación del egreso de la LAFYD

\begin{tabular}{lcc}
\hline \multicolumn{1}{c}{ CoMPETENCIA / ÁrEA } & $\begin{array}{c}\text { HABILIDADES } \\
\text { POR EVALUAR }\end{array}$ & $\begin{array}{c}\text { N DE ÍTEMS EN } \\
\text { EL EXAMEN }\end{array}$ \\
\hline $\begin{array}{l}\text { Evaluación y aplicación de procedimientos pedagógicos } \\
\text { y metodológicos de la actividad física y deporte }\end{array}$ & 9 & 26 \\
$\begin{array}{l}\text { Aplicación de la currícula oficial de educación física en la } \\
\text { educación básica mediante los programas educativos } \\
\text { del área física }\end{array}$ & 14 & 38 \\
$\begin{array}{l}\text { Diseño de programas de actividad física, rehabilitación y } \\
\text { de recreación }\end{array}$ & 11 & 31 \\
$\begin{array}{l}\text { Formulación de organizaciones de actividad física y } \\
\text { deporte }\end{array}$ & 4 & 11 \\
$\begin{array}{l}\text { Evaluación del funcionamiento y adaptación del } \\
\text { individuo a estímulos }\end{array}$ & 4 & 11 \\
\hline Total & 42 & 117 \\
\hline Fuente: Elaboración propia. & & \\
\hline
\end{tabular}

Fuente: Elaboración propia.

2. Revisión de las especificaciones de ítems: Una vez establecido el objeto por evaluar mediante este instrumento, el siguiente paso consistió en revisar que las Acciones y Habilidades definidas cumplieran con la intención de medición de las competencias establecidas en el perfil de egreso. Este proceso se fundamenta en la necesidad de validez del marco de evaluación (García et al., 2017). A través de un proceso colegiado, 15 docentes de la FD de los tres campus valoraron la pertinencia y suficiencia de las Habilidades enlistadas en el paso previo.

3. Elaboración-validación de ítems: este proceso se apoyó en la impartición de talleres en los que participaron 45 docentes de los tres campus de la FD, pertenecientes a las diversas áreas de especialización del programa, con el fin de aprovechar su experiencia en la definición de un instrumento que reflejara la formación que ellos mismos promueven, ahora bajo un marco común.

El insumo principal para el desarrollo de reactivos no fue solo la estructura del examen, sino que se consideró el uso de formatos de especificaciones de reactivos; es decir, un formato que facilitara la identificación de habilidades por medir, junto con el nivel 
cognitivo esperado para cada Habilidad. Con el uso de los formatos de especificaciones se aseguraba que todos los reactivos por incluir en la prueba contarán con las características necesarias para inferir que los puntajes obtenidos realmente eran reflejo de las competencias esperadas en el perfil de egreso. En estos formatos, se presentaba una lista de materias obligatorias y optativas que abonaban a cada área del examen.

En congruencia con el Modelo Educativo de la UABC (UABC, 2013), el diseño de reactivos se orientó hacia la medición de competencias, por lo que se privilegió el planteamiento de casos prácticos, a fin de que el estudiante recurriera a las habilidades y los conocimientos adquiridos a lo largo de su formación universitaria para responder el examen y evitar, en medida de lo posible, reactivos con contenido teórico en los que se apelara a la memorización de definiciones, conceptos o teorías. Una guía para ello fue el uso del nivel cognoscitivo de Bloom Revisado. El desarrollo de reactivos requirió una serie de talleres presenciales en los tres campus de la FD entre noviembre y diciembre del año 2017; adicionalmente, se requirió trabajo a distancia para retroalimentar técnicamente los reactivos generados.

Como ejercicio final de este comité, tras la elaboración de reactivos se realizaron reuniones académicas para que los docentes participantes validarán el trabajo desarrollado. Los docentes del campus Ensenada validaban aquellos reactivos desarrollados por los académicos de Mexicali; a su vez, los profesores de Tijuana validaban los realizados por los docentes de Ensenada; mientras quienes estaban en Mexicali validaban los reactivos elaborados en Tijuana.

En cada campus las actividades se distribuían en cinco mesas de trabajo, una para cada una de las áreas de la prueba. En cada mesa había, al menos, tres docentes de la FD que valoraban la pertinencia de los reactivos realizados por sus colegas de otro campus. Esta dinámica permitió no solo verificar la validez de los reactivos, sino asegurar su aplicación entre campus, ya que los docentes podían asegurar la aplicabilidad de los reactivos realizados en campus distintos a los que ellos participan. Como resultado del proceso de elaboración-validación de reactivos, se conformó un instrumento con 164 reactivos listos para pilotear. Todos estos reactivos fueron desarrollados por los docentes de la FD y validados por la misma comunidad académica.

\section{b) Cuestionario para el estudiante}

Aunado a la aplicación de la prueba, se aplicó un cuestionario enfocado en conocer las competencias intra e interpersonales que, de acuerdo con la literatura, influyen en resultados de la formación profesional. En este sentido, el diseño del instrumento se orientó hacia la valoración de aspectos, como: dedicación, sentido de pertenencia a la institución, autoeficacia académica, intereses, valores sociales y visión positiva de sí mismos (National Academies of Sciences, Engineering and Medicine, 2017). Cabe destacar, que este instrumento fue revisado por los actores educativos de la FD, en particular por los gestores del Programa de la LAFYD.

A partir de la propuesta de la National Academies of Sciences, Engineering and Medicine (2017), complementada con las investigaciones de Blanco y otros (2016), Dugan (2015), Paunesku y otros (2015) y Renninger y Hidhi (2006), el cuestionario se conformó según aparece en el cuadro 2. 
Cuadro 2. Dimensiones e indicadores que conforman el cuestionario de estudiantes para la evaluación del egreso de la LAFYD

\begin{tabular}{ll}
\hline \multicolumn{1}{c}{ DiMENSIÓN } & \multicolumn{1}{c}{ INDICADORES } \\
\hline \multirow{2}{*}{ Dedicación } & Autocontrol \\
& Responsabilidad \\
& Trabajo duro \\
& Persistencia \\
& Establecimiento de metas \\
\hline Sentido de pertenencia a la & Integración y adaptación a la UABC \\
institución & Integración y adaptación a la FD \\
\hline \multirow{2}{*}{ Autoeficacia académica } & Comunicación \\
& Atención \\
\hline Mentalidad de crecimiento & Comprensión \\
\hline Objetivos de utilidad y valores & Orientación hacia la excelencia \\
\hline Objetivos intrínsecos e intereses & Percepción de competencia académica \\
\hline & Percepción de competencia personal \\
\hline Metas prosociales y valores & Orientación al logro \\
\hline Fuente: Elaboración de formación en contenidos disciplinares \\
\hline
\end{tabular}

El cuestionario no pretendió establecer un esquema de factores asociados al resultado en el primero de los instrumentos, sino que se esquematizó de esta manera con el fin de contar con información adicional al logro de aprendizaje respecto a aquellos elementos que, de acuerdo con los autores revisados, son relevantes para una formación profesional exitosa. Si bien el objetivo del cuestionario no se centra en poner a prueba a los estudiantes, su perspectiva sobre las dimensiones consideradas será de gran valía para la retroalimentación de la formación ofrecida en la LAFYD.

Las dimensiones del cuestionario se incluyeron con el fin de valorar aspectos de dedicación, pertenencia a la institución y diversos niveles de orientación de los esfuerzos de los estudiantes. Esta decisión se basó en la intención de contar con información que permitiera valorar si los egresados contaban con habilidades para regular sus propias acciones hacia un éxito académico (National Academies of Sciences, Engineering and Medicine, 2017), incluyendo también su perspectiva sobre su nivel de integración y adaptación a la universidad y a la facultad que los formó profesionalmente. Además de conocer los conocimientos y habilidades en relación con el perfil de egreso, la información del cuestionario permitiría valorar otros elementos de los egresados que pueden considerarse como relevantes para orientar un egreso exitoso.

\section{c) Proceso de aplicación piloto}

Posterior a los procesos antes descritos, se llevó a cabo una prueba piloto con el objetivo de valorar las condiciones de aplicación, así como el comportamiento psicométrico de los reactivos de ambos instrumentos. Esta labor recayó fuertemente en los agentes del IIDE, dada su condición de asesoría metodológica; sin embargo, como se ha mencionado, las 
cuestiones técnicas no se establecieron como el eje del proyecto, sino como una herramienta que soportara las decisiones sobre los instrumentos diseñados con evidencias de los participantes en la prueba piloto. Bajo esa dinámica, los resultados psicométricos que más adelante se presentan respaldan los resultados de los estudiantes, más no consideran un papel protagónico en el proyecto.

Con la intención de contar con una aplicación dinámica de los instrumentos, así como apegarse a lo señalado en el Programa Cero Residuos 4 , establecido por la UABC en 2018, la FD decidió que esta evaluación debía aplicarse mediante un formato computarizado. Si bien hay posturas que cuestionan la posibilidad del uso de este medio en instrumentos a gran escala, dado su posible sesgo en la medición de habilidades concretas (Jiménez, 2018), la población estudiantil de la LAFYD hace uso constante de medios tecnológicos para el desarrollo de sus actividades formativas ${ }^{5}$, cuestión que sustentó la decisión del uso de esta estrategia de administración de instrumentos. La interfaz electrónica fue desarrollada por un consultor técnico externo a la universidad bajo la supervisión del IIDE.

La aplicación se realizó en salas de cómputo de los tres campus a un total de 193 participantes, entre estudiantes de último semestre y egresados. La aplicación se realizó del 20 al 26 de septiembre de 2018. De los 193 participantes, 91 (47,2\%) de ellos estudiaron en el campus Tijuana, 33 (17,1\%) en el campus Ensenada y $69(35,8 \%)$ en Mexicali.

El método utilizado, si bien enfocado en la construcción de dos instrumentos, resalta la participación colegiada de los docentes del programa, asegurando no solo que los instrumentos construidos fueran acordes con el perfil de egreso esperado (y, en consecuencia, obtener una retroalimentación con base en la formación estipulada formalmente en los tres campus), sino que también reflejan las posturas de quienes interactúan día a día con los estudiantes. Esto se fundamenta en el hecho que el marco conceptual de la evaluación fue desagregado por completo por los docentes y validado por ellos mismos. Adicionalmente, el uso de un medio computarizado para la aplicación de los instrumentos va acorde con la política ambiental de la universidad, con la intención formativa en el uso de las TIC y en agilizar el proceso de recolección de información.

\section{Resultados}

\subsection{Resultados psicométricos}

Los resultados psicométricos son aquellos indicadores cuantitativos que orientan la solidez de las interpretaciones de los resultados, mismos que se muestran diferenciados por cada uno de los instrumentos utilizados.

\section{a) Prueba para valorar los conocimientos y habilidades de los egresados}

Si bien el enfoque de evaluación utilizado se fundamenta en una postura comprensiva (Stake, 2006) no considera el uso de instrumentos de medición como nocivo, solo como insuficiente en sí mismo para describir cualquier realidad, los resultados psicométricos de

\footnotetext{
4. Orientada al manejo responsable de recursos para las funciones de la universidad. Mayor información en: http://gaceta.uabc.mx/tags/cero-residuos

${ }^{5}$ Esto se asegura gracias a que una de las asignaturas obligatorias en la formación básica del programa lleva por título “Tecnologías de la Información y Comunicación”, en la cual los estudiantes practican el uso de herramientas informáticas. La PUA puede consultarse en: http://deportes.uabc.mx/images/UABC/plan2012/OBasicas/PUA-Tecnologias-de-laInformacion-y-la-Comunicacion.pdf
} 
los instrumentos no deben valorarse como definitorios del instrumento en sí, sino como un medio de referencia (siempre volátil) de una realidad en particular. En ese sentido, este ejercicio resulta una primera aproximación a la construcción de un instrumento que necesariamente debe ser reajustado con cada aplicación, considerando que los valores estimados dependen de la población que dio respuesta a la prueba.

Se consideraron los psicométricos universalmente aceptados para estimar la fiabilidad y validez de los 164 reactivos aplicados. Para esta valoración se hizo uso de la Teoría Clásica de los Test (TCT), debido a que la baja población que presentó la prueba (193 sustentantes) no favorece el uso de otra perspectiva. A pesar de esto, el uso de TCT ofrece la posibilidad de identificar las características de los reactivos y, con ello, definir cuáles de ellos deben ser usados para emitir calificación. Para este proceso se calcularon niveles de dificultad, así como de correlación punto-biserial como elementos determinantes de la calidad psicométrica de los reactivos. Estos dos indicadores son lo que regularmente se consideran como mínimos para valorar la calidad de medida de cada reactivo. El proceso de calibración consistió en dos análisis, estableciendo como base una correlación puntobiserial positiva como medio de decisión de inclusión de cada reactivo con fines de emisión de calificación. Si bien el estándar utilizado puede ser discutido para instrumentos a gran escala, la consideración de una primera aplicación, así como el nivel bajo de respuestas justificó la necesidad de ampliar el espectro de calibración psicométrica (cuadro 3).

Cuadro 3. Resumen de características psicométricas de la prueba para valorar el ejercicio profesional de la LAFYD

\begin{tabular}{lc}
\hline \multicolumn{1}{c}{ ELEMENTO } & VALOR \\
\hline Participantes evaluados & 193 \\
Reactivos piloteados & 164 \\
Reactivos con parámetros aceptables & 148 \\
Media de aciertos & 78,40 \\
Error típico de la media & 0,96 \\
Varianza & 176,76 \\
Desviación típica & 13,30 \\
Facilidad media & 0,53 \\
Correlación punto biserial media & 0,20 \\
Fiabilidad (Alfa de Cronbach) & 0,83 \\
Error típico de medida & 5,44 \\
\hline Fue: Elabrion
\end{tabular}

Fuente: Elaboración propia.

Vale mencionar que no hubo respuestas omitidas, dado que el sistema informático de la administración de la prueba no permitía concluir el ejercicio a menos que se diera una respuesta a cada uno de los reactivos. Como puede valorarse en el cuadro 3, el resultante de reactivos con parámetros aceptables fue de 148, por lo que solo se prescindió de 16 reactivos (9.7\% del total piloteados). La pérdida de reactivos debido a valores psicométricos no deseados se considera baja para un ejercicio de estas características; su inclusión hubiera sido contraproducente respecto a lo requerido para la emisión de calificación. Por su parte, el promedio de dificultad es medio, además que la correlación punto-biserial media es aceptable; lo mismo sucede con el Alfa de Cronbach.

La variabilidad de puntajes es alta, ello se explica en función del rango de respuestas resultantes. No se consideró un método de calibración excluyente de sustentantes atípicos con fines de mejora de indicadores psicométricos, en virtud que se considera que las 
respuestas bajas atípicas también son parte de la realidad que el ejercicio evaluativo pretende mostrar.

Como puede observarse en este resumen de psicométricos bajo la TCT, los 148 reactivos tienen características favorables para emitir una calificación de los sustentantes. Ahora, considerando la estructura deseada, particularmente el número de reactivos estimado por los docentes de la FD para emitir calificación, solo se consideraron 106 reactivos. Los reactivos seleccionados para calificar fueron aquellos con mejores resultados psicométricos en lo individual. Los 42 reactivos restantes se consideran parte de un pool que la FD puede usar a voluntad, aunque no fueron utilizados para emitir calificación en esta ocasión.

Cabe mencionar las limitantes psicométricas del instrumento. En función de la baja cantidad de sustentantes, es necesario valorar la posibilidad de acumular más aplicaciones, con fines de tener una mayor cantidad de respuestas que favorezcan hacer estimaciones más robustas a través de la Teoría de Respuesta al ítem (TRI) (Martínez, 2001), así como el desarrollo de análisis factoriales que ayuden a la valoración de evidencias de validez de constructo del instrumento.

\section{b) Cuestionario para el estudiante}

Al igual que la prueba, el cuestionario requiere un análisis que nos permita valorar sus características métricas. El cuestionario, de la misma manera que el otro instrumento, tiene una limitación importante dado el número de sustentantes que lo contestaron. El número de sustentantes no permite el desarrollo de análisis factoriales para identificar la estructura de las dimensiones que lo conforman (y con ello verificar empíricamente el modelo de áreas por evaluar definidas por la comunidad académica de la FD), y en caso de calcularse, podría resultar en que los factores observados no puedan ser valorados en ejercicios de réplica, lo cual daría indicios de poca estabilidad de medida del instrumento. Para poder realizar un análisis de este tipo se esperaría tener 10 veces más de sustentantes respecto al número de variables del cuestionario (Nunally, 1972; Thorndike, 1982). El cuestionario aplicado cuenta con 38 variables en total (sin contar datos generales de los estudiantes); para la estimación de un análisis factorial exploratorio se requeriría, al menos, 380 sustentantes. Por otra parte, no se considera pertinente valorar el desarrollo de un análisis factorial confirmatorio, debido a que el nivel de medición de las variables es mayoritariamente ordinal. Independientemente de esta condición, la fiabilidad general del cuestionario mostró un resultado de .663 en el Alfa de Cronbach. Este valor podría haber aumentado con la eliminación de algunas variables, sin embargo, no se consideró la posibilidad de eliminación de variable alguna, debido al interés por su valoración por parte de la comunidad académica.

$\mathrm{Al}$ igual que con la prueba, se sugiere acumular respuestas de más aplicaciones, con el fin de valorar la estructura factorial del instrumento, lo cual permitiría hacer inferencias más precisas respecto a sus evidencias de validez.

\subsection{Resultados de los sustentantes}

La metodología utilizada en este proyecto se fundamentó en procesos psicométricos aceptados universalmente, aunque es el enfoque de construcción de una realidad en particular bajo los actores mismos es lo que permite darles sentido a los puntajes generados. Para la delimitación de los resultados es preciso indicar primeramente la 
metodología para la emisión de valores de los estudiantes en la prueba para valorar los conocimientos y habilidades de los egresados de la LAFYD.

a) Definición de puntos de corte

Con el fin de poder emitir un puntaje de los estudiantes y dar un sentido interpretativo a los datos brutos generados por la prueba para valorar el ejercicio profesional de la LAFYD, la FD estableció la necesidad de contar con un método que les permitiera hacer una valoración criterial del desempeño de sus estudiantes. Si bien bajo una perspectiva comprensiva el uso de método normativos parece más adecuado, dado que refleja la esencia misma de la estimación de una realidad momentánea (Jiménez, 2016), el personal directivo de la FD estableció la necesidad de contar con una referencia criterial, dados sus intereses particulares. En coincidencia con el enfoque comprensivo, se consideró pertinente que el método para la definición de puntos de corte de la prueba debiera ser aquel que permitiera la consideración del juicio de la comunidad académica de la FD.

Se consideró el uso del método Angoff modificado (Cizek, 2012; Jornet et al., 2011 ; Ricker, 2006). Este método se basa en la consideración del juicio experto de la comunidad académica para la determinación del mínimo esperado en cada uno de los reactivos de la prueba. El método Angoff establece como eje la idea del sustentante mínimamente competente; implica que cada uno de los especialistas valore en lo individual el porcentaje mínimo de estudiantes que respondería correctamente a cada uno de los reactivos de la prueba. Valorando el contenido de cada reactivo, cada juez estableció en una plantilla el porcentaje mínimo de estudiantes mínimamente competentes que podría contestarlo correctamente. Una vez teniendo la estimación de cada especialista sobre cada reactivo, se calcularon las medias de proporciones definidas, el resultado fungió como el porcentaje de aciertos mínimo requerido por área para considerarse competente. El proceso se realizó de manera iterativa hasta que los académicos participantes establecieron un punto de corte concluyente.

Cuadro 4. Punto de corte acordado para cada una de las áreas de la prueba

\begin{tabular}{ccc}
\hline \multirow{2}{*}{ Área } & $\begin{array}{c}\text { PUNTO DE CORTE ACORdado, } \\
\text { EXPRESADO EN PORCENTAJE DE ACIERTOS }\end{array}$ & $\begin{array}{c}\text { PUNTO DE CORTE ACORDADO, } \\
\text { EXPRESADO EN NÚMERO DE ACIERTOS* }\end{array}$ \\
\hline 1 & 59,16 & 14 \\
2 & 49,92 & 16 \\
3 & 76,42 & 21 \\
4 & 72,59 & 8 \\
5 & 68,93 & 8 \\
\hline Nota: * Debido a que las estimaciones de los jueces se realizaron en términos relativos, el \\
equivalente en número de aciertos se presenta redondeado. \\
Fuente: Elaboración propia.
\end{tabular}

Con la participación de 15 docentes de los tres campus de la FD, el 31 de octubre de 2018 se realizó la reunión de puntos de corte en las instalaciones del IIDE. En la reunión, los docentes definieron el mínimo de desempeño esperado para cada reactivo en cada una de las áreas de la prueba. Como resultado del proceso, el porcentaje de aciertos mínimo para cada área de la prueba aparece en el cuadro 4.

Los porcentajes establecidos por la comunidad académica de la LAFYD representan los mínimos esperados para valorar la competencia de los sustentantes en la prueba definida. Estos puntos de corte se mantendrán vigentes hasta que la comunidad decida hacer algún 
ajuste sobre ellos. Por el momento, estos valores representan la media de valoraciones realizadas por los especialistas de los tres campus de la FD respecto a los reactivos que conformaron el instrumento.

Adicionalmente, la FD esperaba la delimitación de niveles de desempeño en función de los resultados de los sustentantes en cada una de las áreas de la prueba, con la finalidad de obtener un resultado global por cada persona. Al respecto, y considerando que cada área de la prueba está delimitada por un número distinto de reactivos, no se consideró pertinente la construcción de un indicador cuantitativo que conjuntara la diversidad de puntajes, sino que se estableció una decisión en función de la cantidad de áreas en las cuales se superó el punto de corte.

El resultado global de los estudiantes se estableció con fines interpretativos, resaltando alcances y limitaciones en función de las evidencias métricas de los reactivos utilizados, como lo indican los estándares requeridos para estos fines (AERA, 2014). En ese sentido, las condiciones establecidas por el grupo de expertos para el otorgamiento de un resultado global en particular se muestran en el cuadro 5.

Cuadro 5. Áreas que se requieren para la obtención de un resultado global en particular

\begin{tabular}{lc}
\hline \multicolumn{1}{c}{ NIVEL DE DESEMPEÑO } & ÁREAS REQUERIDAS \\
\hline Insuficiente & 1 ó 2 \\
Suficiente & 3 ó 4 \\
Destacado & 5 \\
\hline
\end{tabular}

Fuente: Elaboración propia.

Como se indica en el cuadro 5, de acuerdo con el estándar establecido por la comunidad académica de la FD, es necesario que los participantes pasen el punto de corte de al menos tres áreas para considerar un desempeño Suficiente, se considerará Destacado a quien pase los puntos de corte de las cinco áreas que conforman la prueba.

Estas decisiones permitieron la emisión de puntajes por estudiante, que, a su vez, facilitó el desarrollo de estadísticos descriptivos que reflejan el desempeño de los estudiantes y egresados de la LAFYD respecto a las competencias establecidas en el perfil de egreso. Cabe considerar las limitaciones de los resultados que a continuación se muestran. En concordancia con el enfoque de evaluación seleccionado, los resultados son reflejo de la construcción de una realidad en particular, delimitada por los reactivos seleccionados para calificar y los contenidos que éstos representan. Los resultados no pueden interpretarse como una valía de toda la complejidad que representa una formación profesional. Consideran únicamente los contenidos medidos y no toda la posibilidad de ejercicio profesional que otorga la LAFYD; en consecuencia, los resultados deben complementarse con valoraciones adicionales, como el promedio total de calificaciones, así como los resultados de aprendizaje mostrados en las diversas asignaturas que no son abarcadas en la prueba, como acciones de actividad física, por ejemplo. Asimismo, cabe recalcar que los estándares utilizados no son per se, sino que responden a los reactivos utilizados, así como a los juicios de los especialistas.

b) Resultados generales y por campus

A continuación se muestra el porcentaje de estudiantes en cada una de las categorías de desempeño global (figura 1) 


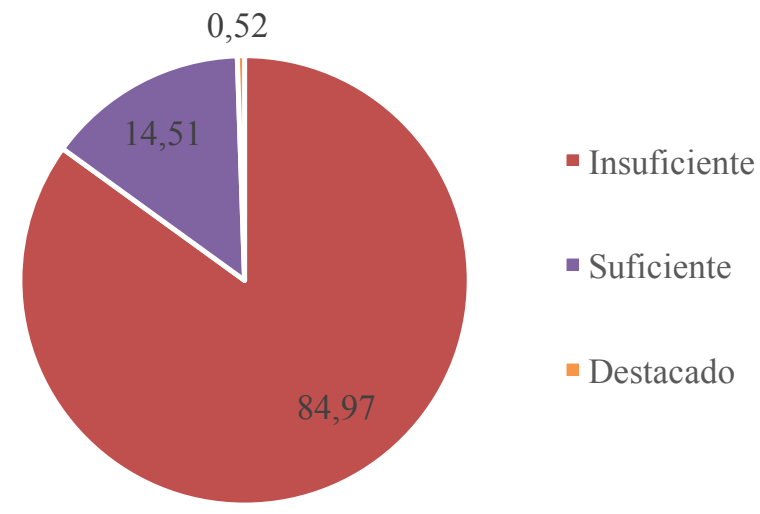

Figura 1. Porcentaje de estudiantes por categorías globales de desempeño Fuente: Elaboración propia.

Como se puede observar, la mayor parte de los participantes no alcanza a obtener un nivel de suficiencia bajo los estándares establecidos por la comunidad académica de la FD. Solo una persona obtuvo un resultado Destacado, logrando pasar el punto de corte en las cinco áreas de la prueba (figura 2).

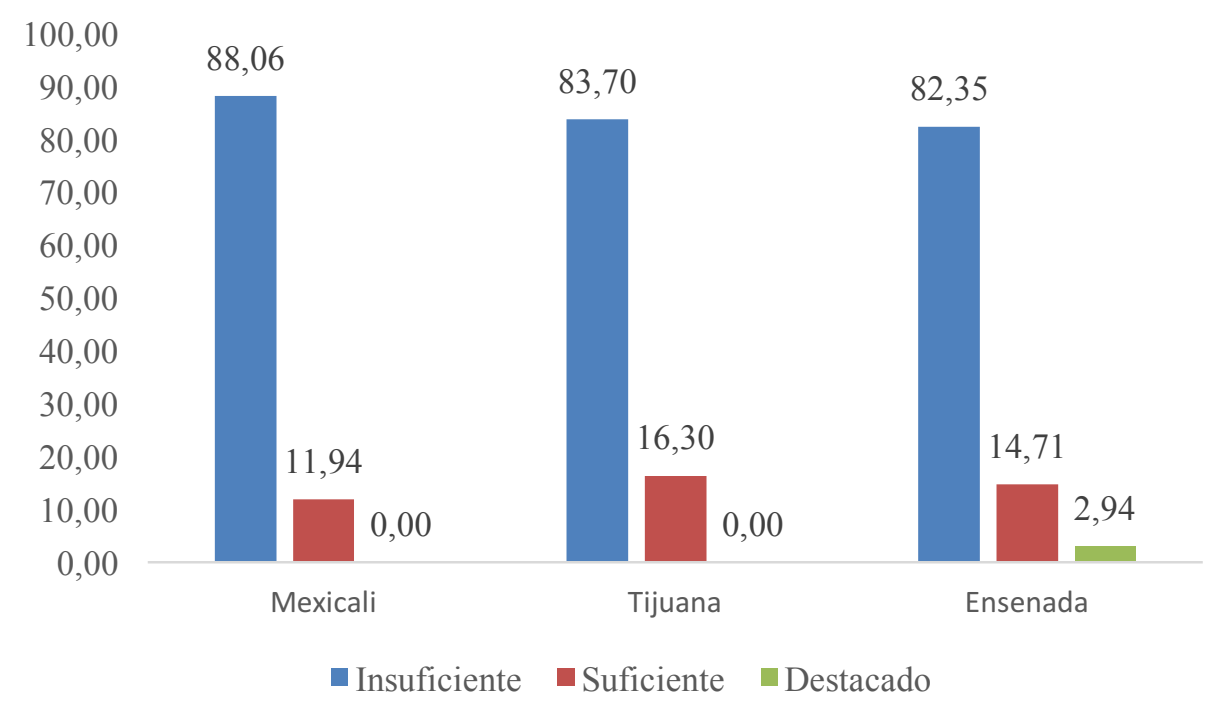

Figura 2. Porcentaje de estudiantes por categorías globales de desempeño por campus Fuente: Elaboración propia.

La distribución de sustentantes por nivel de desempeño por campus nos permite observar cómo los resultados son similares entre cada uno de los campus de la FD. En general, la distribución de participantes entre niveles de desempeño definidos por la comunidad académica se mantiene similar, resaltan Tijuana y Ensenada, en el primero de éstos hay una mayor proporción de estudiantes en nivel Suficiente, mientras que el segundo es el único campus con un estudiante destacado.

Cabe considerar que, de los 193 sustentantes, 78 de ellos (40,41\%) tenían una condición de egresados, mientras que 115 (59,58\%) eran estudiantes de octavo semestre. Los resultados diferenciados por condición de participante se muestran a continuación (figura $3)$. 


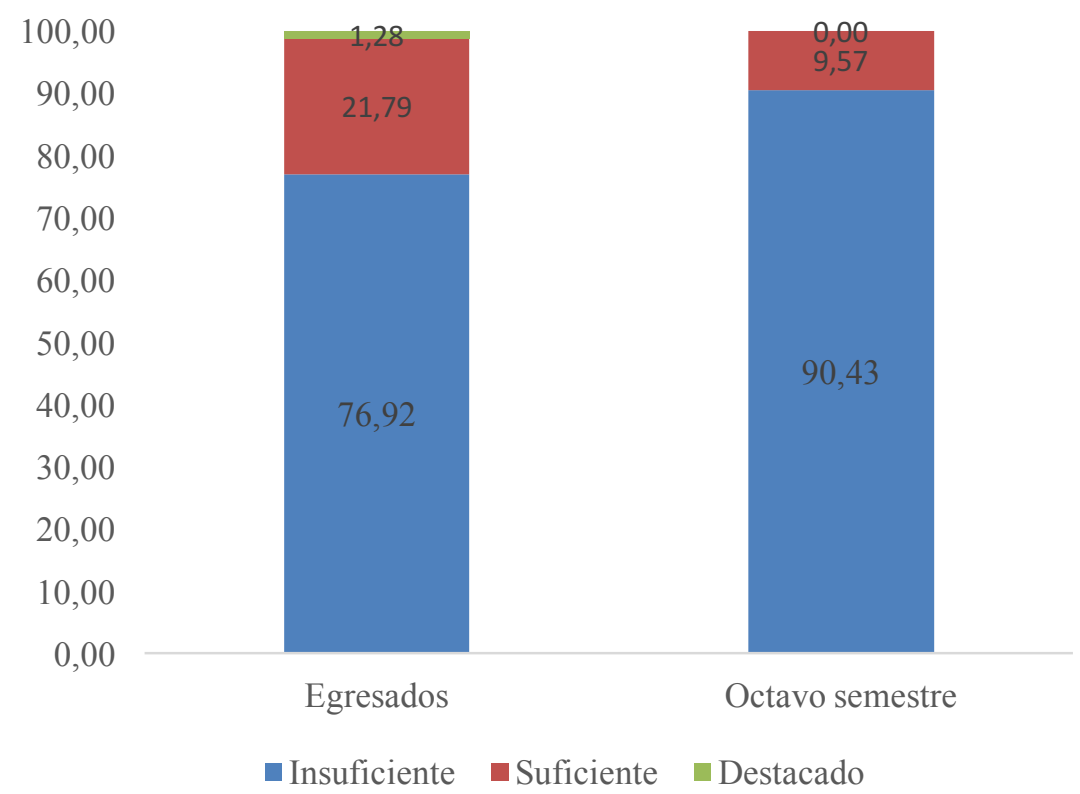

Figura 3. Porcentaje de estudiantes por categorías globales de desempeño por condición Fuente: Elaboración propia.

Los participantes en condición de egresados muestran claramente un mejor resultado respecto a quienes se encontraban aún en condición de estudiantes de octavo semestre. Si bien puede requerir más estudios a profundidad, este resultado puede relacionarse con la experiencia profesional que adquieren quienes egresan del programa. Si bien más adelante se muestran resultados del cuestionario para estudiantes, vale mostrar el cruce de resultados de los sustentantes respecto al área de interés de ejercicio profesional manifestada.

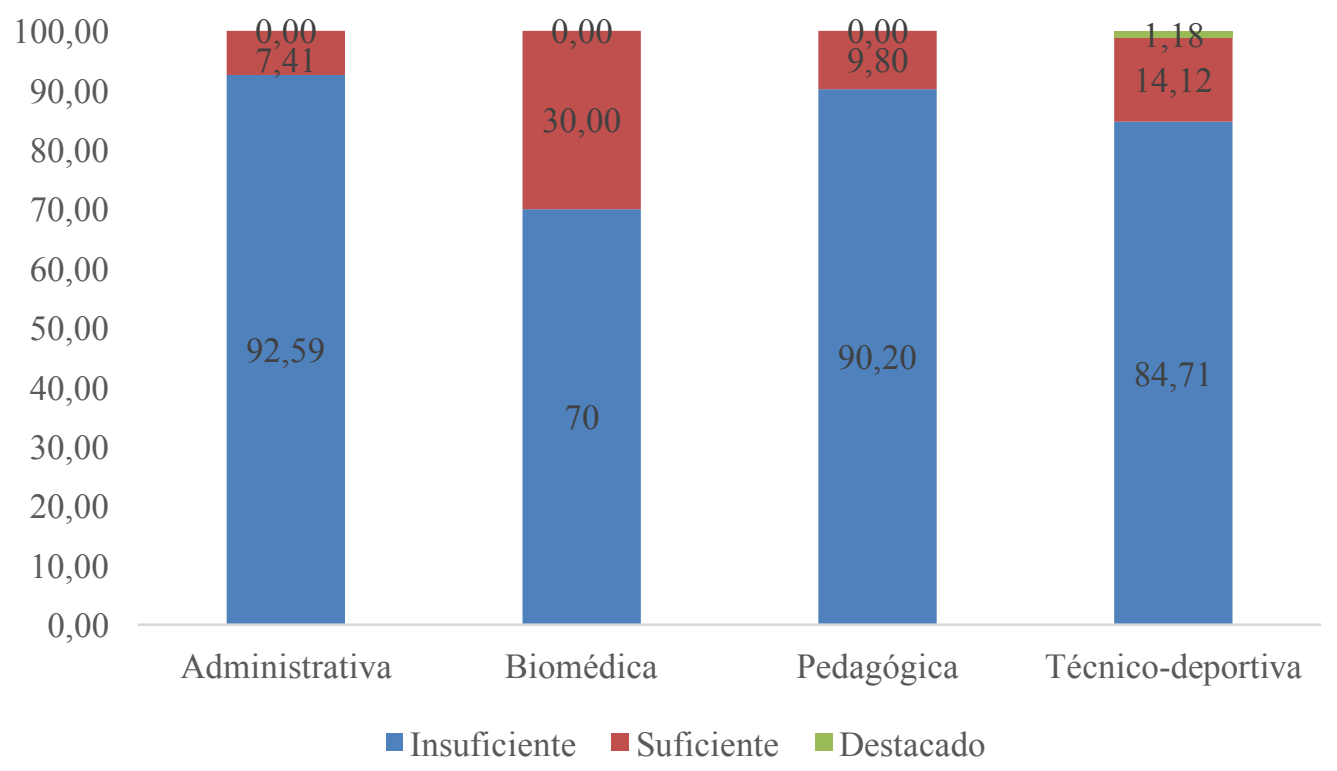

Figura 4. Porcentaje de estudiantes por categorías globales de desempeño respecto al área de interés de ejercicio profesional

Fuente: Elaboración propia. 
La figura 4 muestra los resultados globales en función del área de interés del ejercicio profesional de los sustentantes. Claramente se observa que quienes buscan el área biomédica como elemento de ejercicio profesional tienen mayor proporción de resultados en la categoría de Suficiente.

Respecto a los resultados por área de la prueba, están fuertemente relacionados con los puntos de corte establecidos por la comunidad académica de la FD. Como se observa en la figura 5, para valorar el desempeño de los sustentantes se considera el criterio del punto de corte como referente.

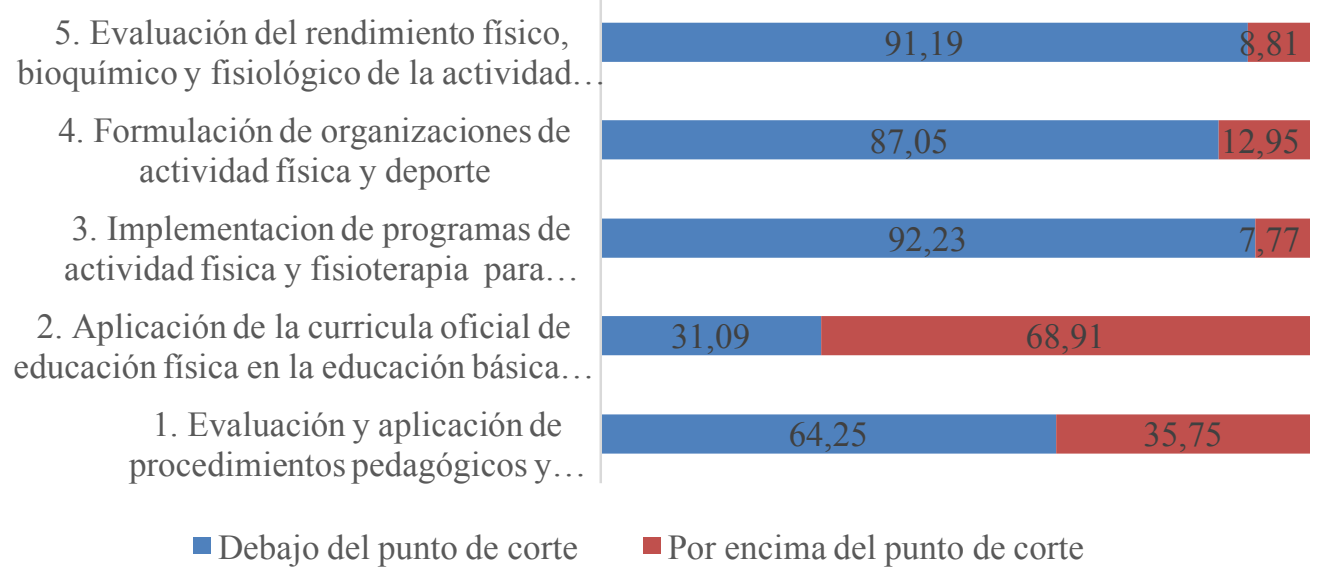

Figura 5. Porcentaje de estudiantes debajo y por encima del punto de corte de cada área de la prueba.

Fuente: Elaboración propia.

La figura 5 muestra cómo las áreas 1 y 2 de la prueba son las que permitieron un mayor porcentaje de estudiantes por encima del punto de corte, mientras que el resto presentaron un estándar un tanto más estricto. Esta decisión se dio en función de los mínimos considerados dentro de cada mesa de trabajo, dados los reactivos utilizados para emitir calificación.

Los resultados de la prueba utilizada para conocer las habilidades de los egresados de la LAFYD reflejan como una gran proporción de sustentantes de los tres campus no alcanzan el estándar mínimo esperado en las cinco áreas de la prueba. Considerando que el desarrollo del marco de evaluación tomó en cuenta las habilidades más representativas de las competencias del perfil de egreso (siempre y cuando pudieran valorarse bajo una prueba de opción múltiple), es necesario hacer una revisión de los logros de aprendizaje manifiestos por los estudiantes en su formación profesional. Vale considerar la posibilidad de establecer formas de valoración y retroalimentación del aprendizaje durante la formación profesional que aseguren el acercamiento del desempeño de estudiantes al ideal establecido en el perfil de egreso del plan de estudios de la LAFYD.

Los resultados de aprendizaje mostrados por los egresados y estudiantes participantes se comparan respecto a la realidad del programa de la LAFYD construida por la comunidad académica de la FD en sus tres campus. El estándar mínimo establecido por los académicos es poco alcanzable para la mayoría de los participantes. En una valoración criterial como la construida es factible valorar los procesos formativos planteados desde el plan de estudios, así como la operación del programa. Si bien una evaluación de estas características no permite el desarrollo de inferencias sobre el programa educativo en sí, 
los resultados reflejados son un insumo importante de retroalimentación de los fundamentos del programa.

\section{c) Resultados del cuestionario del estudiante}

El enfoque de evaluación utilizado no considera a la prueba como supraordenada al ejercicio de valoración de los resultados del cuestionario; en ese sentido, no se consideró un esquema de análisis de factores asociados al logro. En consecuencia, se decidió la valoración de algunas categorías relacionadas con lo que la literatura suele relacionar con un egreso exitoso de nivel licenciatura (National Academies of Sciences, Engineering and Medicine, 2017). Los resultados son realmente extensos, además que, con el pendiente de desarrollar análisis factoriales que permitan delinear de manera más precisa las dimensiones planteadas, por el momento solo se presentan resultados de algunos reactivos que se consideraron más importantes para fines de divulgación (figura 6).

Si bien la mayor parte de los sustentantes se manifiesta en desacuerdo con haberse rendido frente a tareas complejas, no debe dejarse de lado que $21.2 \%$ de los participantes manifestó un determinado grado de acuerdo respecto a haberse rendido. La posibilidad de rendirse frente a tareas complejas refleja un foco importante en la formación en actividad física y deporte, dada el perfil generalmente competitivo de quienes la estudian (figura 7).

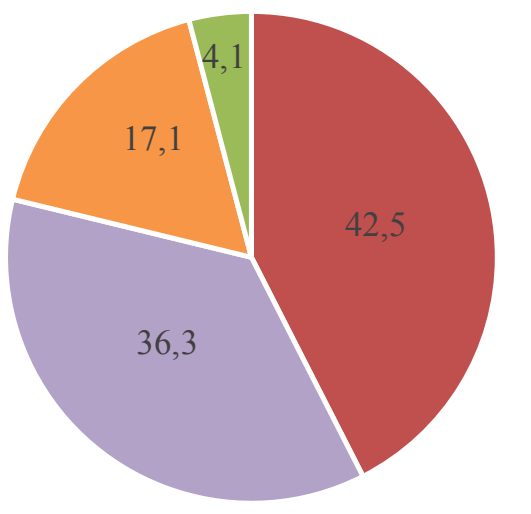

- Totalmente en desacuerdo

En desacuerdo

- De acuerdo

- Totalmente de acuerdo

Figura 6. Durante la mayor parte de mi formación universitaria llegué a rendirme al realizar algunas actividades y tareas complejas

Fuente: Elaboración propia.

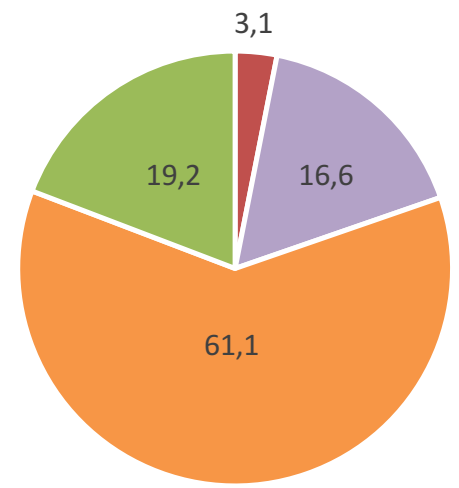

- Totalmente en desacuerdo

- En desacuerdo

- De acuerdo

- Totalmente de acuerdo

Figura 7. Durante mis estudios universitarios fui parte importante de la UABC Fuente: Elaboración propia. 
Un alto porcentaje de los participantes manifestaron haberse sentido como una parte importante dentro de la UABC durante sus estudios universitarios; sin embargo, un porcentaje considerable de sustentantes (en este caso, 19.7\%) manifiestan un grado de desacuerdo respecto a esta cuestión. Este porcentaje no debe desestimarse, aun cuando no representa la opinión de la mayoría de los encuestados.

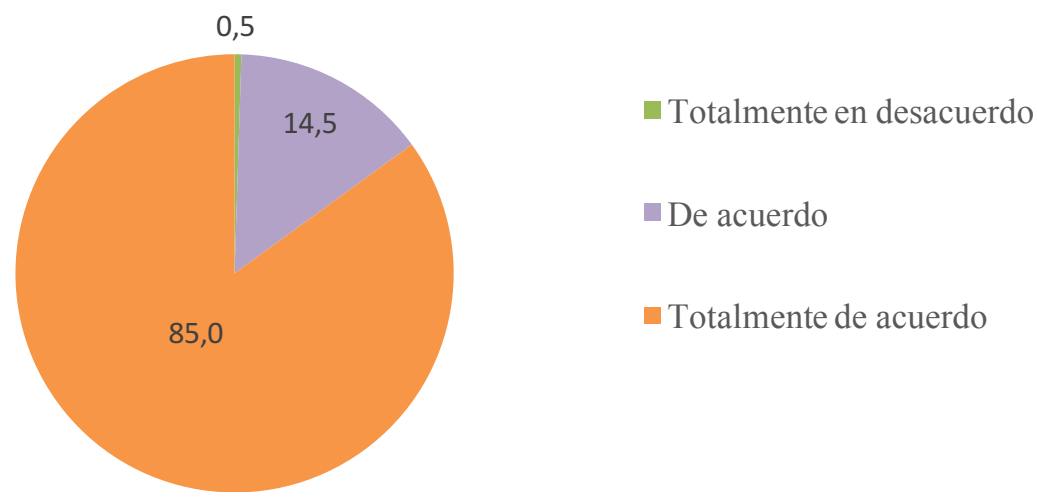

Figura 8. A partir de mi formación universitaria siento que soy capaz de seguir aprendiendo cosas nuevas para cada vez ser un(a) mejor Licenciado(a) en Actividad Física y Deportes

Fuente: Elaboración propia.

Prácticamente la totalidad de los participantes en la evaluación considera un grado de acuerdo respecto a que la formación recibida les permitió seguir aprendiendo cosas nuevas. Esto es, sin duda, positivo para el programa, que fomenta la posibilidad de continuar el desarrollo como egresado de esta formación.

5. Evaluación del funcionamiento y adaptación del individuo a estímulos

4. Formulación de organizaciones de actividad física y deporte

3. Diseño de programas de actividad física, rehabilitación y de recreación

2. Aplicación de la curricula oficial de educación física en la educación básica.

1. Evaluación y aplicación de procedimientos pedagógicos y metodológicos de la...

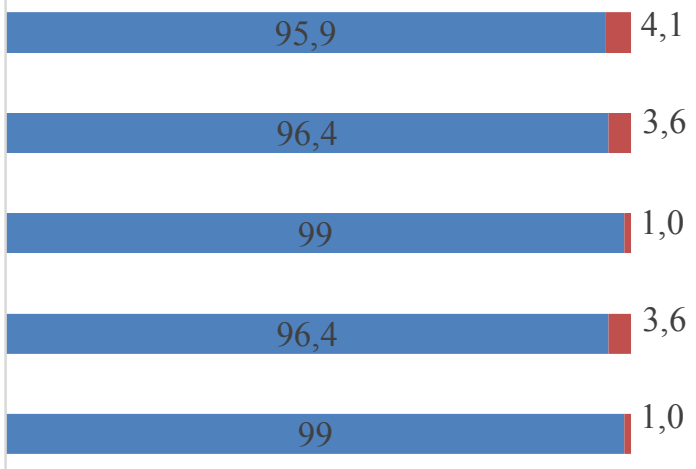

- Sí $\quad$ No

Figura 9. Utilidad de las áreas de la prueba para la práctica profesional Fuente: Elaboración propia.

La dimensión de objetivos intrínsecos e intereses se enfocó fuertemente en la identificación de la valoración de la utilidad y el gusto para dedicarse a las áreas de la prueba (las cuales engloban determinadas competencias establecidas en el perfil de egreso). En el caso de la utilidad, la figura 9 muestra cómo los sustentantes en lo general tienen una alta perspectiva de utilidad de las áreas evaluadas en la prueba. 
5. Evaluación del funcionamiento y adaptación del individuo a estímulos

4. Formulación de organizaciones de actividad física y deporte

3. Diseño de programas de actividad física, rehabilitación y de recreación

2. Aplicación de la curricula oficial de educación física en la educación básica.

1. Evaluación y aplicación de procedimientos pedagógicos y metodológicos de la...

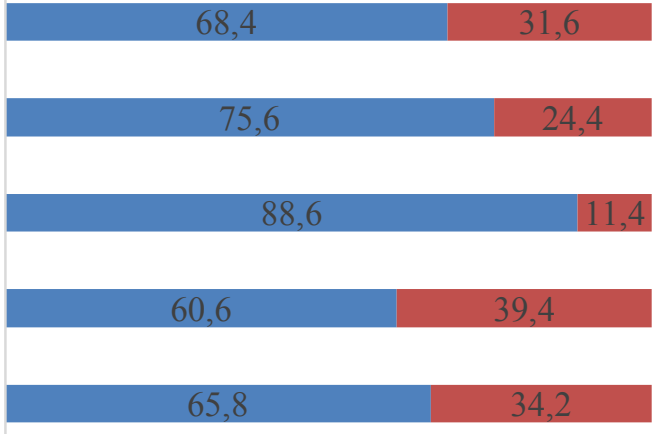

- Sí No

Figura 10. Gusto para dedicarse a cada una de las áreas de la prueba para la práctica profesional

Fuente: Elaboración propia.

Si bien los sustentantes reconocen la utilidad de las áreas de competencia del egresado de la LAFYD de la UABC, el gusto para dedicarse a cada una de ellas es muy diverso. La de mayor aceptación en este sentido es el área de "Diseño de programas de actividad física, rehabilitación y de recreación”, en contraste, la de menor aceptación es el área “Aplicación de la currícula oficial de educación física en educación básica mediante los programas educativos del área física”.

50

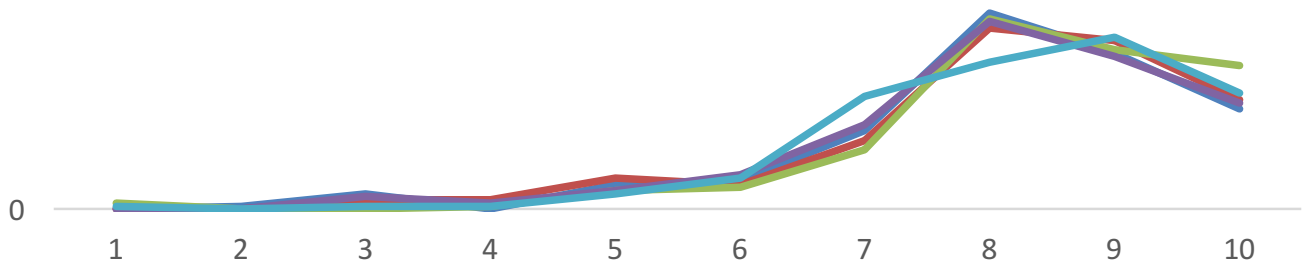

1. Evaluación y aplicación de procedimientos pedagógicos y metodológicos de la actividad física y deporte

-2. Aplicación de la curricula oficial de educación física en la educación básica mediante los programas educativos del área física

3. Diseño de programas de actividad física, rehabilitación y de recreación

4. Formulación de organizaciones de actividad física y deporte

Figura 11. Califica qué tan preparado te sientes para ejercer cada una de las áreas de ejercicio profesional

Fuente: Elaboración propia.

La figura 11 muestra la calificación que los sustentantes dan respecto a cómo valoran su preparación para ejercer cada una de las cinco áreas evaluadas en la prueba. Las áreas siguen una tendencia similar, salvo el caso del área 5 (Evaluación del funcionamiento y adaptación del individuo a estímulos), que pareciera que, en general, se sienten menos preparados respecto al resto de las áreas. Este último juicio de los sustentantes no es menor, incluso valdría un ejercicio de comparación respecto a los resultados de la prueba. 
De acuerdo con los sustentantes, se sienten preparados para el ejercicio profesional, sin embargo, una baja proporción de ellos logran superar el punto de corte establecido por la comunidad académica de la FD en los reactivos utilizados para calificar.

\section{Discusión y conclusiones}

El desarrollo de la evaluación del egreso de la LAFYD es un ejercicio inédito dentro de la UABC. La intención no se reducía únicamente a la elaboración de un instrumento para medir habilidades y conocimientos de determinadas asignaturas del plan de estudios, sino que se pretendía retratar una realidad en particular del egreso en los tres campus en los cuales se ofrece este programa (Stake, 2006). En ese sentido, la participación de la comunidad académica de la FD en el ejercicio permitió establecer resultados de sus sustentantes acordes con la formación esperada por el programa educativo.

Este manuscrito comunicó la metodología utilizada para el cumplimiento del objetivo de esta evaluación, así como las características psicométricas básicas de ambos instrumentos utilizados. Cabe recalcar las limitaciones de evidencias de validez del cuestionario y de la prueba, resultante del bajo número de sustentantes participantes, lo cual imposibilita emitir valores más sólidos en esta primera aplicación. Es necesario considerar la acumulación de información de futuros sustentantes, con el fin de estimar valores más precisos respecto a ambos instrumentos. A pesar de ello, se reconoce que la mayor proporción de instrumentos utilizados tiene amplios niveles de confiabilidad, incluso a pesar de las limitaciones del número de la población utilizada para su estimación. Al igual que cualquier otro instrumento a gran escala, es menester valorar la posibilidad de asegurar su mejora conforme a cada aplicación que se tenga contemplada, buscando, incluso realizar análisis más robustos, como los basados en TRI o en ecuaciones estructurales.

A pesar de esta cuestión, la alta participación de la comunidad de la FD permitió asegurar que los resultados observados en este informe ofrecen una clara posibilidad de contrastar lo que los sustentantes reflejan dada la formación esperada en el programa de la LAFYD de la UABC.

Respecto a los resultados, los puntos de corte establecidos por la comunidad académica de la FD permitieron identificar que altas proporciones de estudiantes no están alcanzando los niveles esperados al terminar su formación. Como sugerencia, es posible considerar el listado de Habilidades marcados en la estructura definida y orientar posibles formas didácticas intra e inter campus para favorecer que los próximos egresados alcancen los niveles esperados por la comunidad de docentes, los cuales, a su vez, devienen de las competencias establecidas en el perfil de egreso. Los resultados en términos de Habilidades muestran una clara necesidad de orientar una formación relacionada con la resolución de problemas propios de la formación, considerando que fue ésta la orientación declarada en los reactivos utilizados para calificar.

Considerando las categorías globales de desempeño, es relevante trabajar con el colectivo docente la implicación que solo $0.52 \%$ de los egresados alcance un nivel Destacado. Por supuesto, se espera que solo algunos estudiantes alcancen un nivel de dominio amplio sobre todas las áreas evaluadas; sin embargo, el alto porcentaje de estudiantes en la categoría de Insuficiente implica necesariamente una reflexión con la comunidad académica, ya que los estudiantes demuestran que no cuentan con habilidades mínimas 
establecidas por la propia comunidad académica en función del perfil de egreso del programa educativo. Pareciera que la condición de egresado favorece a los resultados obtenidos; en ese sentido, ¿de qué manera la experiencia profesional influye en el dominio del perfil de egreso esperado? Si se indaga más en ese sentido pudieran orientarse acciones formativas más cercanas hacia la experiencia profesional, ya que, al menos en este primer ejercicio, esa experiencia parece favorecer a quienes han abandonado las aulas de la FD.

En otro sentido, los resultados entre campus son bastante similares. La FD deberá hacer un ejercicio de reflexión respecto a condiciones de formación en cada campus. Considerando que el plan de estudios que se implementa en los tres campus es el mismo, ¿existen diferencias respecto a formación de la planta docente y las actividades didácticas entre campus? ¿Las condiciones de infraestructura son similares? Si hubiera alguna diferencia de planta, infraestructura o actividades didácticas entre campus, ¿qué elementos nos ayudan a conocer el por qué los estudiantes mantienen un desempeño tan similar en tres contextos relativamente independientes?

Por su parte, si bien solo se muestran algunos resultados del cuestionario, éste refleja percepciones relacionadas con la complejidad de haberse dedicado a estudiar y cumplir con mínimos esperados. Vale considerar también las áreas de interés de empleabilidad mostrada por los participantes en relación con los resultados obtenidos en la prueba, los cuales no necesariamente coinciden. El cuestionario puede utilizarse así no como una medida explicativa de los resultados de la prueba, sino como complementaria respecto a la perspectiva de los sustentantes respecto a la formación recibida en la FD. Por supuesto, como se ha mencionado, esto es solo un panorama inicial, ya que las limitaciones psicométricas del cuestionario requieren ejercicios de aplicación que favorezcan la robustez de la información.

Este ejercicio de evaluación no solo ha permitido identificar el nivel de desempeño de los sustentantes de la FD respecto a un mínimo establecido en función del perfil de egreso, sino que funge como un ejercicio inédito dentro de la UABC, mostrando la posibilidad que una facultad logre establecer esfuerzos en común por parte de su comunidad académica para alcanzar un objetivo unificado. Asimismo, resultó ser un ejercicio importante para la formación en actividad física, al ser el primer trabajo en el país de su tipo. En términos metodológicos, resultó ser una actividad importante bajo un enfoque comprensivo, que tradicionalmente no es la perspectiva evaluativa que define este tipo de evaluaciones.

\section{Agradecimientos}

Este trabajo se realizó y se pudo concluir gracias a la participación de más de 70 docentes de la Facultad de Deportes de la Universidad Autónoma de Baja California, así como al esfuerzo de los estudiantes y egresados participantes.

\section{Referencias}

AERA. (2014). Standards for educational and psychological testing. Washington, DC: American Educational Research Association.

Cizek, G. (2012). Establecimiento de estándares. En S. Downing y T. Haladyna (Comps.), Manual para el desarrollo de pruebas a gran escala (pp. 345-390). Ciudad de México: Ceneval. 
Blanco, H., Aguirre, J., Barrón, J. y Blanco, R. (2016). Composición factorial de la escala de autoeficacia académica en universitarios mexicanos. Formación Universitaria, 9(2), 81-88. https://doi.org/10.4067/So7 18-50062016000200009

Dugan, J. (2015). The measurement of socially responsible leadership: Considerations in establishing psychometric rigor. Educational, Cultural and Psychological Studies, 12, 23-42. https://doi.org/10.7358/ecps-2015-012-duga

García, A., Martínez, F., Cordero, G. y Caso, J. (2017). Evolución del concepto de validez en la medición educativa. En E. Luna y G. Cordero (Coords.), Contribuciones a la evaluación educativa desde la formación doctoral (pp. 15-46). Guadalajara: UdeG/UABC.

Jiménez, A. (2014, mayo). Evaluación a gran escala: Su desarrollo e inconvenientes en su modelo teórico. Comunicación presentada en el Congreso Internacional de Educación: Evaluación. Universidad Autónoma de Tlaxcala.

Jiménez, A. (2016). Evaluación a gran escala del egreso de la licenciatura: Hacia un modelo comprensivo. Tesis doctoral. Universidad Nacional Autónoma de México.

Jiménez, A. (2017). La evaluación de los egresados de formación profesional en México: Reflejo de la implementación de la política de competitividad en la educación superior. Archivos Analíticos de Políticas Educativas, 25(48), 7-23. https://doi.org/10.14507/epaa.25.2868

Jiménez, A. (2018). México en PISA 2015: Desaciertos de su aplicación por medios computarizados. Revista Mexicana de Investigación Educativa, 23(78), 711-731.

Jiménez, A. (en prensa). Reflexiones epistemológicas de la evaluación educativa: Entre el deber ser y lo relativo. Foro de Educación. https://doi.org/10.145 16/fde.636

Jornet, J., González, J., Suárez, J. y Perales, J. (2011). Diseño de procesos de evaluación de competencias: Consideraciones acerca de los estándares en el dominio de las competencias. Bordón, 63(1), 125-145.

Krathwohl, D. (2002). A revision of Bloom's taxonomy: An overview. Theory into Practice, 41(4), 212-2 18. https://doi.org/10.1207/s15430421tip4104_2

Martínez, F. (2001). Evaluación educativa y pruebas estandarizadas. Elementos para enriquecer el debate. Revista de la Educación Superior, 30(4), 71-85.

Mateo, J. (2006). Claves para el diseño de un nuevo marco conceptual para le medición y evaluación educativas. Revista de Investigación Educativa, 24(1), 165-186.

National Academies of Sciences, Engineering and Medicine. (2017). Supporting students' college success. The role of assessment of intrapersonal and interpersonal competences. Washington, DC: The National Academies Press.

Paunesku, D., Walton, G., Romero, C., Smith, E., Yeager, D. y Dweck, C. (2015). Mind-set interventions are a scalable treatment for academic underachievement. Psychological Science, 26(6), 784-793. https://doi.org/10.1177/0956797615571017

Renninger, A. y Hidhi, S. (2006). Revisiting the conceptualization, measurement, and generation of interest. Educational Psychologist, 46(3), 168-184. https://doi.org/10.1080/00461520.2011.587723

Ricker, K. (2006). Setting cut-scores: A critical review of the Angoff and modified Angoff methods. The Alberta Journal of Educational Research, 5(1), 53-64.

Santos Guerra, M. (1988). Patología general de la evaluación educativa. Infancia y Aprendizaje, 41, 143-158. https://doi.org/10.1080/02 103702.1988.10822197

SEP. (2018). Instituciones de educación superior. Recuperado de http://www.ses.sep.gob.mx/instituciones.html 
Stake, R. (2006). Evaluación comprensiva y evaluación basada en estándares. Barcelona: Graó.

Thorndike, L. (1982). Applied psychometrics. Boston, MA: Houghton-Mifflin.

Universidad Autónoma de Baja California (UABC). (2013). Modelo educativo de la UABC. Mexicali: UABC.

Universidad Autónoma de Baja California (UABC). (2015). Plan de desarrollo institucional 20152019. Mexicali: UABC.

Universidad Autónoma de Baja California (UABC). (2017). Perfil de egreso de la licenciatura en actividad física y deportes. Mexicali: UABC.

Universidad Autónoma de Baja California (UABC). (2018). Misión UABC. Mexicali: UABC.

Webb, N. (2012). Identificación del contenido para las pruebas de logro de estudiante. En S. Downing y T. Haladyna (Comps.), Manual para el desarrollo de pruebas a gran escala (pp. 245284). Ciudad de México: Ceneval.

\section{Breve CV de los autores}

\section{José Alfonso Jiménez Moreno}

Doctor en pedagogía por la Universidad Nacional Autónoma de México, es Licenciado en Psicología. Miembro del Sistema Nacional de Investigadores en el nivel Candidato. Trabajó en el Centro Nacional de Evaluación para la Educación Superior (Ceneval) como Coordinador del Examen General para el Egreso de la Licenciatura (EGEL). Fue subdirector de pruebas internacionales en el Instituto Nacional para la Evaluación de la Educación (INEE). Actualmente se desempeña como investigador en el Instituto de Investigación y Desarrollo Educativo de la Universidad Autónoma de Baja California. ORCID ID: https://orcid.org/O000-0003-0704-7883. Email: jose.alfonso.jimenez.moreno@uabc.edu.mx

\section{Juan Pablo Machado Parra}

Licenciado en Actividad Física y Deportes en Universidad Autónoma de Baja California (UABC), es maestro en Innovación e Investigación en Ciencias de la Actividad Física y Deporte (Universidad de León, España) y actualmente estudiante de Doctorado en Ciencias del Ejercicio por la Universidad de León, España. Trabajó en el Instituto del Deporte y de la Cultura Física del estado de Baja California (INDE) como metodologo de los deportes de conjunto. Actualmente se desempeña como Profesor de Tiempo Completo (PTC), así como Coordinador de Formación Basica en la Facultad de Deportes Campus Ensenada en la Universidad Autónoma de Baja California. ORCID ID: https://orcid.org/oooo-0003-2687-6257. Email: machado.juan@uabc.edu.mx

\section{Joaquín Caso Niebla}

Doctor en Psicología Educativa por la Facultad de Psicología de la UNAM. Como parte de su experiencia profesional destaca haber fungido como Coordinador de la Unidad de Evaluación Educativa del Instituto de Investigación y Desarrollo Educativo de la Universidad Autónoma de Baja California y Coordinación del Centro de Orientación Educativa (COE) de la UNAM. Se desempeña como Director del Instituto de Investigación y Desarrollo Educativo de la UABC, miembro del Sistema Nacional de Investigadores. Entre los proyectos de Evaluación de la educación básica en Baja 
California e Intervención Preventiva en contextos educativos. Es miembro de la Sociedad Mexicana de Psicología desde 1995, en el que ha ocupado los cargos Vicepresidente (20052008) y Presidente (2009 a 2013). ORCID ID: https://orcid.org/o000-0002-3557-1722. Email: jcaso@uabc.edu.mx

\section{Emilio Manuel Arrayales Millán}

Licenciado en Ingeniería Industrial y de Sistemas, es maestro en Administración de la Educación Física, Deporte y Recreación y estudiante de doctorado en Ciencias del Deporte en la Universidad de Extremadura, España. Trabajó en el Instituto Tecnológico de Sonora como entrenador y coordinador del deporte selectivo. Trabajó como profesor y entrenador en la Escuela de Deportes de la Universidad Autónoma de Baja California (UABC), subdirector de la Escuela de Deportes de UABC. Actualmente se desempeña como Director y profesor de licenciatura y maestría de la Facultad de Deportes de UABC. ORCID ID: https://orcid.org/oooo-0002-9335-3635. Email: earrayales@uabc.edu.mx 